\title{
Correction to: Arthroscopic primary repair of proximal anterior cruciate ligament tears seems safe but higher level of evidence is needed: a systematic review and meta-analysis of recent literature
}

Jelle P. van der List ${ }^{1,2,3}$ (D) $\cdot$ Harmen D. Vermeijden ${ }^{1,3} \cdot$ Inger N. Sierevelt $^{1,2} \cdot$ Gregory S. DiFelice $^{3} \cdot$ Arthur van Noort $^{1}$. Gino M. M. J. Kerkhoffs ${ }^{2,4,5}$

Published online: 6 November 2019

(c) The Author(s) 2019

\section{Correction to: \\ Knee Surgery, Sports Traumatology, Arthroscopy \\ https://doi.org/10.1007/s00167-019-05697-8}

Unfortunately, the original publication of this article contains an error in table 1 . The revised version of Table 1 is updated here. The original article has been corrected.

The original article can be found online at https://doi.org/10.1007/ s00167-019-05697-8.

Jelle P. van der List

jpjvanderlistmd@gmail.com

1 Department of Orthopaedic Surgery, Spaarne Gasthuis Hospital, Hoofddorp, The Netherlands

2 Amsterdam UMC, Department of Orthopaedic Surgery, University of Amsterdam, Amsterdam, The Netherlands

3 Hospital for Special Surgery, Department of Orthopaedic Surgery, New York, USA

4 Amsterdam UMC, Academic Center for Evidence Based Sports Medicine (ACES), University of Amsterdam, Amsterdam, The Netherlands

5 Amsterdam UMC, Amsterdam Collaboration On Health and Safety in Sports (ACHSS), University of Amsterdam, IOC Research Center, Amsterdam, The Netherlands 
Table 1 Quality assessment of the included studies using the Methodological Index for Non-Randomized Studies (MINORS) criteria

\begin{tabular}{|c|c|c|c|c|c|c|c|c|c|c|c|c|c|}
\hline Authors & Year & Journal/meeting & Evidence & Study design & 1 & 2 & 3 & 4 & 5 & 6 & 7 & 8 & Total \\
\hline Achtnich et al. [1] & 2016 & Arthroscopy & III & Prospective & 2 & 2 & 1 & 2 & 1 & 2 & 2 & 0 & 12 \\
\hline Ateschrang et al. [2] & 2017 & KSSTA & IV & Case series & 2 & 2 & 2 & 2 & 0 & 1 & 1 & 0 & 10 \\
\hline Büchler et al. [11] & 2016 & Knee & IV & Case series & 2 & 2 & 1 & 2 & 0 & 1 & 2 & 0 & 10 \\
\hline Häberli et al. [25] & 2018 & Knee & IV & Case series & 2 & 2 & 1 & 2 & 0 & 2 & 2 & 0 & 11 \\
\hline Heusdens et al. [31] & 2018 & KSSTA & IV & Case series & 2 & 2 & 2 & 1 & 0 & 1 & 2 & 1 & 11 \\
\hline Hoffmann et al. [33] & 2017 & J Orthop Surg Res & IV & Case series & 2 & 2 & 0 & 2 & 1 & 2 & 2 & 0 & 11 \\
\hline Hoogeslag et al. [35] & 2019 & Am J Sports Med & I & RCT & 2 & 2 & 2 & 2 & 1 & 2 & 2 & 2 & 15 \\
\hline Jonkergouw et al. [38] & 2018 & KSSTA & III & Retrospective & 2 & 2 & 1 & 2 & 0 & 1 & 2 & 0 & 10 \\
\hline Kohl et al. [41] & 2016 & $\mathrm{BJJ}$ & IV & Case series & 1 & 2 & 2 & 2 & 0 & 2 & 2 & 0 & 11 \\
\hline Krismer et al. [43] & 2017 & KSSTA & $\mathrm{IV}^{\mathrm{a}}$ & Case series & 2 & 2 & 0 & 2 & 0 & 2 & 2 & 0 & 10 \\
\hline Meister et al. [52] & 2017 & KSSTA & IV & Case series & 2 & 1 & 2 & 2 & 0 & 1 & 2 & 0 & 10 \\
\hline Mukhopadhyay et al. [54] & 2018 & Chin J Traumatol & IV & Case series & 1 & 2 & 2 & 2 & 0 & 2 & 2 & 0 & 11 \\
\hline Osti et al. [62] & 2019 & KSSTA & IV & Case series & 2 & 2 & 2 & 1 & 0 & 1 & 2 & 0 & 10 \\
\hline
\end{tabular}

Only the non-comparative part of the MINORS criteria was used (i.e. first 8 questions). The criteria of MINORS [70] with 0 points when not reported, 1 when reported but not adequate, and 2 when reported and adequate. Maximum score is 16

1. A clearly stated aim: the question addressed should be precise and relevant in the light of available literature

2. Inclusion of consecutive patients: all patients potentially fit for inclusion (satisfying the criteria for inclusion) have been included in the study during the study period (no exclusion or details about the reasons for exclusion)

3. Prospective collection of data: data were collected according to a protocol established before the beginning of the study

4. End points appropriate to the aim of the study: unambiguous explanation of the criteria used to evaluate the main outcome which should be in accordance with the question addressed by the study. In addition, the end points should be assessed on an intention-to-treat basis

5. Unbiased assessment of the study end point: blind evaluation of objective end points and double-blind evaluation of subjective end points. Otherwise, the reasons for not blinding should be stated

6. Follow-up period appropriate to the aim of the study: the follow-up should be sufficiently long to allow the assessment of the main endpoint and possible adverse events

7. Loss to follow-up less than 5\%: all patients should be included in the follow-up. Otherwise, the proportion lost to follow-up should not exceed the proportion experiencing the major end point

8. Prospective calculation of the study size: information of the size of detectable difference of interest with a calculation of $95 \%$ CI, according to the expected incidence of the outcome event, and information about the level for statistical

${ }^{\text {a }}$ This study reported being a level II study but we have classified this case series with failure analysis as level IV study

Open Access This article is distributed under the terms of the Creative Commons Attribution 4.0 International License (http://creativecommons.org/licenses/by/4.0/), which permits unrestricted use, distribution, and reproduction in any medium, provided you give appropriate credit to the original author(s) and the source, provide a link to the Creative Commons license, and indicate if changes were made. 